HEPHY-PUB 805/05

hep-th/0507281

July 2005

\title{
INSTANTANEOUS BETHE-SALPETER EQUATION WITH EXACT PROPAGATORS
}

\author{
Wolfgang LUCHA* \\ Institut für Hochenergiephysik, \\ Österreichische Akademie der Wissenschaften, \\ Nikolsdorfergasse 18, A-1050 Wien, Austria \\ Franz F. SCHÖBERL ${ }^{\dagger}$ \\ Institut für Theoretische Physik, Universität Wien, \\ Boltzmanngasse 5, A-1090 Wien, Austria
}

\begin{abstract}
Consequent application of the instantaneous approximation to both the interaction and all propagators of the bound-state constituents allows us to forge, within the framework of the Bethe-Salpeter formalism for the description of bound states, an instantaneous form of the Bethe-Salpeter equation with exact (i.e., full) propagators of the bound-state constituents. This instantaneous equation generalizes the well-known Salpeter equation the derivation of which needs the additional assumption of free propagation of the bound-state constituents.
\end{abstract}

PACS numbers: 11.10.St, 03.65.Ge, 03.65.Pm

* E-mail address: wolfgang.lucha@oeaw.ac.at

$\dagger$ E-mail address: franz.schoeberl@univie.ac.at 


\section{Introduction}

Within relativistic quantum field theories, the appropriate framework, and a standard tool, for the description of bound states from first principles is the Bethe-Salpeter formalism [1]. In order to circumvent its complexity and problems of interpretation of (all) its solutions, a simplification of the Bethe-Salpeter equation has been sought and soon found: the Salpeter equation, obtained by a three-dimensional reduction [2]. Since then, this equation has been frequently employed to study, e.g., the phenomenology of mesons as bound states of quarks $[3,4]$ either entirely numerically [5-22] or analytically to the utmost possible extent [23-26].

The Bethe-Salpeter equation reduces to Salpeter's equation under two assumptions [2]:

- Every bound-state constituent propagates as a free particle with some effective mass; this constant should adequately parametrize all dynamical self-energy contributions.

- All interactions between the particles which constitute or form the bound state under investigation are instantaneous in the center-of-momentum frame of the bound state. (Lorentz covariance is achieved by requiring all interactions to depend, in momentum space, only on those components of the relative momenta of the involved bound-state constituents which are perpendicular to the total momentum of this bound state [8].)

However, the above assumption of free propagators for the bound-state constituents faces a serious conceptual problem [3,27-32], which provides an argument strongly in favour of the use of the corresponding exact propagators. In quantum field theory, the Dyson-Schwinger equations connect these propagators, which are 2-point Green functions, and the particular $n$-point Green functions that represent the interactions in the Bethe-Salpeter equation. As consequence of this, the propagators and interactions cannot be chosen independently from each other. Accordingly, in non-Abelian gauge theories, such as quantum chromodynamics, the simultaneous assumptions of free-particle propagation of bound-state constituents and confining interaction, induced by quantum chromodynamics, are intrinsically inconsistent. In spite of this, apart from the study of the spectroscopy of light mesons in Ref. [7] all of the above investigations adhere to the free-propagator approximation from the very beginning.

In view of this, we demonstrate in this note that the free-propagator assumption can be easily skipped in our construction of a consistent instantaneous Bethe-Salpeter formalism. We give the resulting relativistic wave equation for exact albeit instantaneous propagators.

\section{Instantaneous Bethe-Salpeter Formalism}

\subsection{Homogeneous Bethe-Salpeter Equation}

By approximating the interactions responsible for the formation of the bound states under consideration by their static limits but retaining - in contrast to the additional assumption adopted in the derivation of the Salpeter equation [2] — the exact form of the propagators of the bound-state constituents, we construct in this section an instantaneous Bethe-Salpeter formalism representing a formal improvement of the Salpeter equation. Our intention is to employ the resulting eigenvalue equation of motion eventually for the description of mesons regarded as bound states of a quark and an antiquark formed by the strong interactions [3]. Accordingly, let us start the present analysis with a brief exposition of the essential features of the Bethe-Salpeter equation for bound states composed of a fermion and an antifermion. 
First of all, we have to recall the standard kinematics used for a system of two particles (labelled $i=1,2$ ) located at coordinates $x_{1}, x_{2}$ and carrying momenta $p_{1}, p_{2}$. Introducing two arbitrary real (dimensionless) parameters $\eta_{1}, \eta_{2}$ satisfying $\eta_{1}+\eta_{2}=1$, we define for this two-particle system the center-of-momentum coordinate $X$ and the relative coordinate $x$, as well as the conjugate variables, the total momentum $P$ and the relative momentum $p$, by

$$
X \equiv \eta_{1} x_{1}+\eta_{2} x_{2}, \quad x \equiv x_{1}-x_{2}, \quad P \equiv p_{1}+p_{2}, \quad p \equiv \eta_{2} p_{1}-\eta_{1} p_{2},
$$

such that $P X+p x=p_{1} x_{1}+p_{2} x_{2}$.

Within the framework of the Bethe-Salpeter formalism, a bound state of momentum $P$ and mass $M_{\mathrm{B}}$, denoted generically by the Hilbert-space vector $|\mathrm{B}(P)\rangle$, is represented by its Bethe-Salpeter amplitude $\Psi$. In the more convenient momentum-space representation, the Bethe-Salpeter amplitude $\Psi(p)$ is defined as Fourier transform of the matrix element of the time-ordered product of the two field operators that describe the bound-state constituents, evaluated with respect to vacuum state $|0\rangle$ and bound state $|\mathrm{B}(P)\rangle$, after factorizing off the center-of-momentum motion. Consequently, suppressing all spinor (or Dirac) and internal indices [as well as the dependence of $\Psi(p)$ on the momentum $P$ of the bound state $|\mathrm{B}(P)\rangle$ ], the momentum-space Bethe-Salpeter amplitude of any fermion-antifermion bound state is

$$
\Psi(p) \equiv \exp (\text { i } P X) \int \mathrm{d}^{4} x \exp (\text { i } p x)\left\langle 0\left|\mathrm{~T}\left(\psi_{1}\left(x_{1}\right) \bar{\psi}_{2}\left(x_{2}\right)\right)\right| \mathrm{B}(P)\right\rangle ;
$$

here it is evidently understood that the field operators $\psi_{1}, \bar{\psi}_{2}$, respectively, are evaluated at

$$
x_{1}=X+\eta_{2} x, \quad x_{2}=X-\eta_{1} x .
$$

The Bethe-Salpeter amplitude $\Psi$ satisfies the formally exact Bethe-Salpeter equation, which involves two dynamical ingredients: the exact (or "full" or "dressed") propagators of the two bound-state constituents and the Bethe-Salpeter interaction kernel $K$. This kernel is a fully amputated 4-point Green function, defined (merely perturbatively!) as the sum of the countable infinity of all Feynman diagrams for two-particle into two-particle scattering. Moreover, it has to be Bethe-Salpeter irreducible, i.e., two-particle irreducible with respect to the two bound-state constituents. In the momentum space the kernel $K(p, q)$ depends on the relative momenta $p$ and $q$ of initial and final state (as well as on the momentum $P$ of the bound state). Denoting the propagator of the fermion $i=1,2$ by $S_{i}(p)$, the Bethe-Salpeter equation for a fermion-antifermion bound state reads, in momentum-space representation,

$$
S_{1}^{-1}\left(p_{1}\right) \Psi(p) S_{2}^{-1}\left(-p_{2}\right)=\frac{\mathrm{i}}{(2 \pi)^{4}} \int \mathrm{d}^{4} q K(p, q) \Psi(q) .
$$

Multiplying Eq. (1) by $S_{1}\left(p_{1}\right)$ from the left and $S_{2}\left(-p_{2}\right)$ from the right, the Bethe-Salpeter equation becomes

$$
\Psi(p)=\frac{\mathrm{i}}{(2 \pi)^{4}} S_{1}\left(p_{1}\right) \int \mathrm{d}^{4} q K(p, q) \Psi(q) S_{2}\left(-p_{2}\right)
$$

\subsection{Instantaneous Approximation}

The instantaneous (or static) approximation to the Bethe-Salpeter formalism is based on the assumption that the Bethe-Salpeter kernel $K$ entering in the Bethe-Salpeter equations (1) or (2) depends only on the spatial components $\boldsymbol{p}$ and $\boldsymbol{q}$ of the relative momenta $p$ and $q$ :

$$
K(p, q)=\hat{K}(\boldsymbol{p}, \boldsymbol{q})
$$


This restriction corresponds to the assumption of an instantaneous interaction between the bound-state constituents and amounts to ignoring all retardation effects in the interaction.

In the instantaneous approximation the Bethe-Salpeter equation may be reduced to an equation of motion for a Salpeter amplitude (or equal-time bound-state wave function) $\Phi$. In momentum-space representation the Salpeter amplitude $\Phi(\boldsymbol{p})$ is obtained by integrating the Bethe-Salpeter amplitude $\Psi(p)$ over the time component $p_{0}$ of the relative momentum:

$$
\Phi(\boldsymbol{p}) \equiv \frac{1}{2 \pi} \int \mathrm{d} p_{0} \Psi(p) .
$$

Introducing, for the sake of notational simplicity, for the interaction term the abbreviation

$$
I(\boldsymbol{p}) \equiv \frac{1}{(2 \pi)^{3}} \int \mathrm{d}^{3} q \hat{K}(\boldsymbol{p}, \boldsymbol{q}) \Phi(\boldsymbol{q})
$$

upon integration over $p_{0}$ the Bethe-Salpeter equation (2) assumes the instantaneous form

$$
\Phi(\boldsymbol{p})=\frac{\mathrm{i}}{2 \pi} \int \mathrm{d} p_{0} S_{1}\left(p_{1}\right) I(\boldsymbol{p}) S_{2}\left(-p_{2}\right) ;
$$

here the momenta $p_{1}, p_{2}$ are, of course, regarded as functions of total and relative momenta:

$$
p_{1}=\eta_{1} P+p, \quad p_{2}=\eta_{2} P-p .
$$

\subsection{Exact Fermion Propagator}

The actual evaluation of the integral over the time component $p_{0}$ of the relative momentum $p$ of the tensor product of fermion propagators in the (integrated) Bethe-Salpeter equation (4) requires the knowledge of the dependence of the (full) fermion propagator $S_{i}(p)$ on $p_{0}$.

The fermion propagator $S_{i}(p)$ is the solution of the fermion Dyson-Schwinger equation, in view of its rôle in all analyses of the phenomenon of dynamical chiral-symmetry breaking also called the gap equation (for reviews of this subject consult, for instance, Refs. [33-39]). By Lorentz covariance (if preserved by gauge fixing) the general solution $S_{i}(p)$ for the exact fermion propagator is represented by only two Lorentz-scalar functions, $M_{i}\left(p^{2}\right)$ and $Z_{i}\left(p^{2}\right)$ :

$$
S_{i}(p)=\frac{\mathrm{i} Z_{i}\left(p^{2}\right)}{\not p-M_{i}\left(p^{2}\right)+\mathrm{i} \varepsilon}, \quad \not p \equiv p^{\mu} \gamma_{\mu} .
$$

Hence, the exact propagator $S_{i}(p)$ is related to the free propagator of a fermion of mass $m$,

$$
S_{0}(p, m)=\frac{\mathrm{i}}{\not p-m+\mathrm{i} \varepsilon} \equiv \mathrm{i} \frac{\not p+m}{p^{2}-m^{2}+\mathrm{i} \varepsilon},
$$

according to $S_{i}(p)=Z_{i}\left(p^{2}\right) S_{0}\left(p, M_{i}\left(p^{2}\right)\right)$. This form of the exact fermion propagators $S_{i}(p)$ is equivalent to an alternate representation in terms of scalar functions $A_{i}\left(p^{2}\right)$ and $B_{i}\left(p^{2}\right)$,

$$
S_{i}(p)=\frac{\mathrm{i}}{A_{i}\left(p^{2}\right) \not p-B_{i}\left(p^{2}\right)+\mathrm{i} \varepsilon}
$$

by means of the identifications

$$
M_{i}\left(p^{2}\right)=\frac{B_{i}\left(p^{2}\right)}{A_{i}\left(p^{2}\right)}, \quad Z_{i}\left(p^{2}\right)=\frac{1}{A_{i}\left(p^{2}\right)} .
$$


Now, in order to continue our present quest for a generalization of the Salpeter equation we have to impose a restriction on the two Lorentz-scalar functions, denoted by $M_{i}\left(p^{2}\right)$ and $Z_{i}\left(p^{2}\right)$ in the representation (5), which characterize each exact fermion propagator $S_{i}(p)$. In full accordance with the spirit of the instantaneous approximation to the interaction kernel entering in the Bethe-Salpeter equation, as well as for the sake of conceptual simplicity, we neglect retardation effects also in the propagators and assume both of the functions $M_{i}\left(p^{2}\right)$ and $Z_{i}\left(p^{2}\right)$ to depend only on the spatial components, $\boldsymbol{p}$, of the involved momentum $p$. This means we make the substitutions $M_{i}\left(p^{2}\right) \rightarrow M_{i}\left(\boldsymbol{p}^{2}\right), Z_{i}\left(p^{2}\right) \rightarrow Z_{i}\left(\boldsymbol{p}^{2}\right)$, which entails for the above-mentioned relation between exact and free propagator $S_{i}(p)=Z_{i}\left(\boldsymbol{p}^{2}\right) S_{0}\left(p, M_{i}\left(\boldsymbol{p}^{2}\right)\right)$. Our (pragmatic) point of view is supported, for example, within quantum chromodynamics by findings for the quark propagator [40-46] obtained from effective Coulomb-gauge model Hamiltonians designed to implement effects of spontaneous breakdown of chiral symmetry. Clearly, our assumption corresponds to the simplest conceivable dependence of $S_{i}(p)$ on $p_{0}$.

\subsection{Exact-Propagator Instantaneous Bethe-Salpeter Formalism}

Upon acceptance of the " $p_{0}^{2}=0$ " approximation for both of the involved propagators in the sense as specified above in Subsect. 2.3, the formulation of the corresponding instantaneous Bethe-Salpeter equation follows a "well-paved road." We introduce the one-particle energy

$$
E_{i}(\boldsymbol{p}) \equiv \sqrt{\boldsymbol{p}^{2}+M_{i}^{2}\left(\boldsymbol{p}^{2}\right)}, \quad i=1,2,
$$

and the generalized Dirac Hamiltonian

$$
H_{i}(\boldsymbol{p}) \equiv \gamma_{0}\left[\boldsymbol{\gamma} \cdot \boldsymbol{p}+M_{i}\left(\boldsymbol{p}^{2}\right)\right], \quad i=1,2,
$$

satisfying $H_{i}^{2}(\boldsymbol{p})=E_{i}^{2}(\boldsymbol{p})$ and $H_{i}(\boldsymbol{p}) \gamma_{0}=\gamma_{0} H_{i}(-\boldsymbol{p})$. With these notions, we define energy projection operators $\Lambda_{i}^{ \pm}(\boldsymbol{p})$ for positive or negative energy $\pm E_{i}(\boldsymbol{p})$ of particle $i, i=1$, 2 , by

$$
\Lambda_{i}^{ \pm}(\boldsymbol{p}) \equiv \frac{E_{i}(\boldsymbol{p}) \pm H_{i}(\boldsymbol{p})}{2 E_{i}(\boldsymbol{p})}, \quad i=1,2
$$

exhibiting all projection-operator properties $\Lambda_{i}^{ \pm}(\boldsymbol{p}) \Lambda_{i}^{ \pm}(\boldsymbol{p})=\Lambda_{i}^{ \pm}(\boldsymbol{p}), \Lambda_{i}^{ \pm}(\boldsymbol{p}) \Lambda_{i}^{\mp}(\boldsymbol{p})=0$ and $\Lambda_{i}^{+}(\boldsymbol{p})+\Lambda_{i}^{-}(\boldsymbol{p})=1$, and satisfying $\Lambda_{i}^{ \pm}(\boldsymbol{p}) \gamma_{0}=\gamma_{0} \Lambda_{i}^{ \pm}(-\boldsymbol{p})$. All of this enters in the identity

$$
\not p+M_{i}\left(\boldsymbol{p}^{2}\right)=\left(\left[p_{0}+E_{i}(\boldsymbol{p})\right] \Lambda_{i}^{+}(\boldsymbol{p})+\left[p_{0}-E_{i}(\boldsymbol{p})\right] \Lambda_{i}^{-}(\boldsymbol{p})\right) \gamma_{0} .
$$

With the help of this identity, the exact fermion propagator $S_{i}(p)$ may be cast into the form

$$
S_{i}(p)=\mathrm{i} Z_{i}\left(\boldsymbol{p}^{2}\right)\left(\frac{\Lambda_{i}^{+}(\boldsymbol{p})}{p_{0}-E_{i}(\boldsymbol{p})+\mathrm{i} \varepsilon}+\frac{\Lambda_{i}^{-}(\boldsymbol{p})}{p_{0}+E_{i}(\boldsymbol{p})-\mathrm{i} \varepsilon}\right) \gamma_{0} .
$$

This is nothing else but the partial fraction decomposition of the right-hand side of Eq. (5).

With the decomposition (7) of the fermion propagators at one's disposal, the evaluation of the integral of the tensor product of fermion propagators in the Bethe-Salpeter equation (4) is straightforward. Employing the residue theorem, a simple contour integration yields

$$
\begin{aligned}
& \int \mathrm{d} p_{0} S_{1}\left(p_{1}\right) \otimes S_{2}\left(-p_{2}\right) \\
& =-2 \pi \mathrm{i} Z_{1}\left(\boldsymbol{p}_{1}^{2}\right) Z_{2}\left(\boldsymbol{p}_{2}^{2}\right)\left(\frac{\Lambda_{1}^{+}\left(\boldsymbol{p}_{1}\right) \gamma_{0} \otimes \gamma_{0} \Lambda_{2}^{-}\left(\boldsymbol{p}_{2}\right)}{P_{0}-E_{1}\left(\boldsymbol{p}_{1}\right)-E_{2}\left(\boldsymbol{p}_{2}\right)}-\frac{\Lambda_{1}^{-}\left(\boldsymbol{p}_{1}\right) \gamma_{0} \otimes \gamma_{0} \Lambda_{2}^{+}\left(\boldsymbol{p}_{2}\right)}{P_{0}+E_{1}\left(\boldsymbol{p}_{1}\right)+E_{2}\left(\boldsymbol{p}_{2}\right)}\right) .
\end{aligned}
$$


By use of this result in Eq. (4), we arrive at our instantaneous Bethe-Salpeter equation for fermion-antifermion bound states, with exact propagators of the bound-state constituents:

$$
\Phi(\boldsymbol{p})=Z_{1}\left(\boldsymbol{p}_{1}^{2}\right) Z_{2}\left(\boldsymbol{p}_{2}^{2}\right)\left(\frac{\Lambda_{1}^{+}\left(\boldsymbol{p}_{1}\right) \gamma_{0} I(\boldsymbol{p}) \gamma_{0} \Lambda_{2}^{-}\left(\boldsymbol{p}_{2}\right)}{P_{0}-E_{1}\left(\boldsymbol{p}_{1}\right)-E_{2}\left(\boldsymbol{p}_{2}\right)}-\frac{\Lambda_{1}^{-}\left(\boldsymbol{p}_{1}\right) \gamma_{0} I(\boldsymbol{p}) \gamma_{0} \Lambda_{2}^{+}\left(\boldsymbol{p}_{2}\right)}{P_{0}+E_{1}\left(\boldsymbol{p}_{1}\right)+E_{2}\left(\boldsymbol{p}_{2}\right)}\right) .
$$

Upon applying to Eq. (8) $\Lambda_{1}^{ \pm}\left(\boldsymbol{p}_{1}\right)$ from the left and $\Lambda_{2}^{ \pm}\left(\boldsymbol{p}_{2}\right)$ from the right, one realizes that precisely half of the components of the Salpeter amplitude $\Phi(\boldsymbol{p})$ have to vanish identically:

$$
\Lambda_{1}^{+}\left(\boldsymbol{p}_{1}\right) \Phi(\boldsymbol{p}) \Lambda_{2}^{+}\left(\boldsymbol{p}_{2}\right)=\Lambda_{1}^{-}\left(\boldsymbol{p}_{1}\right) \Phi(\boldsymbol{p}) \Lambda_{2}^{-}\left(\boldsymbol{p}_{2}\right)=0 .
$$

The difference of these two constraints on the Salpeter amplitude $\Phi(\boldsymbol{p})$ yields

$$
\frac{H_{1}\left(\boldsymbol{p}_{1}\right)}{E_{1}\left(\boldsymbol{p}_{1}\right)} \Phi(\boldsymbol{p})+\Phi(\boldsymbol{p}) \frac{H_{2}\left(\boldsymbol{p}_{2}\right)}{E_{2}\left(\boldsymbol{p}_{2}\right)}=0
$$

\subsection{Formulation as Equivalent Eigenvalue Problem}

Starting already with the pioneering work of Salpeter [2], it has become a common practice to cast the instantaneous Bethe-Salpeter equation into the shape of an eigenvalue problem. Let us perform this step in the present study too: with the help of, for instance, the identity

$\Lambda_{1}^{ \pm}\left(\boldsymbol{p}_{1}\right)\left[H_{1}\left(\boldsymbol{p}_{1}\right) \Phi(\boldsymbol{p})-\Phi(\boldsymbol{p}) H_{2}\left(\boldsymbol{p}_{2}\right)\right] \Lambda_{2}^{\mp}\left(\boldsymbol{p}_{2}\right)= \pm\left[E_{1}\left(\boldsymbol{p}_{1}\right)+E_{2}\left(\boldsymbol{p}_{2}\right)\right] \Lambda_{1}^{ \pm}\left(\boldsymbol{p}_{1}\right) \Phi(\boldsymbol{p}) \Lambda_{2}^{\mp}\left(\boldsymbol{p}_{2}\right)$

which follows from

$$
\Lambda_{i}^{ \pm}(\boldsymbol{p}) H_{i}(\boldsymbol{p})=H_{i}(\boldsymbol{p}) \Lambda_{i}^{ \pm}(\boldsymbol{p})= \pm E_{i}(\boldsymbol{p}) \Lambda_{i}^{ \pm}(\boldsymbol{p}), \quad i=1,2
$$

we easily extract from Eq. (8) our (explicit) eigenvalue equation for the Salpeter amplitude $\Phi(\boldsymbol{p})$, with the (total) energy $P_{0}$ of the bound state $|\mathrm{B}(P)\rangle$ as the corresponding eigenvalue:

$$
\begin{aligned}
& H_{1}\left(\boldsymbol{p}_{1}\right) \Phi(\boldsymbol{p})-\Phi(\boldsymbol{p}) H_{2}\left(\boldsymbol{p}_{2}\right) \\
& +Z_{1}\left(\boldsymbol{p}_{1}^{2}\right) Z_{2}\left(\boldsymbol{p}_{2}^{2}\right)\left[\Lambda_{1}^{+}\left(\boldsymbol{p}_{1}\right) \gamma_{0} I(\boldsymbol{p}) \gamma_{0} \Lambda_{2}^{-}\left(\boldsymbol{p}_{2}\right)-\Lambda_{1}^{-}\left(\boldsymbol{p}_{1}\right) \gamma_{0} I(\boldsymbol{p}) \gamma_{0} \Lambda_{2}^{+}\left(\boldsymbol{p}_{2}\right)\right] \\
& =P_{0} \Phi(\boldsymbol{p})
\end{aligned}
$$

It goes without saying that every solution of the above eigenvalue equation for the Salpeter amplitude $\Phi(\boldsymbol{p})$ has to be still subject to the constraints (9). More precisely, this eigenvalue equation (10) alone is definitely not equivalent to the "true" instantaneous Bethe-Salpeter equation (8). This fact can be revealed by application of the projectors $\Lambda_{1}^{ \pm}\left(\boldsymbol{p}_{1}\right)$ from the left and the projectors $\Lambda_{2}^{ \pm}\left(\boldsymbol{p}_{2}\right)$ from the right to the instantaneous Bethe-Salpeter equation (8) and the eigenvalue equation (10): The $\Lambda_{1}^{ \pm}\left(\boldsymbol{p}_{1}\right) \otimes \Lambda_{2}^{\mp}\left(\boldsymbol{p}_{2}\right)$ components of Eq. (8) and Eq. (10) are certainly identical whereas the $\Lambda_{1}^{ \pm}\left(\boldsymbol{p}_{1}\right) \otimes \Lambda_{2}^{ \pm}\left(\boldsymbol{p}_{2}\right)$ projections yield, for the instantaneous Bethe-Salpeter equation (8), the two constraints (9) but, for the eigenvalue equation (10),

$$
\left\{P_{0} \mp\left[E_{1}\left(\boldsymbol{p}_{1}\right)-E_{2}\left(\boldsymbol{p}_{2}\right)\right]\right\} \Lambda_{1}^{ \pm}\left(\boldsymbol{p}_{1}\right) \Phi(\boldsymbol{p}) \Lambda_{2}^{ \pm}\left(\boldsymbol{p}_{2}\right)=0,
$$

which does not necessarily imply $\Lambda_{1}^{ \pm}\left(\boldsymbol{p}_{1}\right) \Phi(\boldsymbol{p}) \Lambda_{2}^{ \pm}\left(\boldsymbol{p}_{2}\right)=0$. Consequently, equivalence only holds between the instantaneous Bethe-Salpeter equation (8), on the one hand, and the set formed by the eigenvalue equation (10) amended by the constraints (9), on the other hand. 
In the rest frame of the two-particle system under consideration, defined by $\boldsymbol{P}=\mathbf{0}$, the time component of the total momentum $P$ reduces to the bound-state mass eigenvalue $M_{\mathrm{B}}$, that is, $P_{0}=M_{\mathrm{B}}$. The instantaneous Bethe-Salpeter equation, in particular, its form given by the eigenvalue equation (10), then involves only the relative momentum $\boldsymbol{p}=\boldsymbol{p}_{1}=-\boldsymbol{p}_{2}$ :

$$
\begin{aligned}
& H_{1}(\boldsymbol{p}) \Phi(\boldsymbol{p})-\Phi(\boldsymbol{p}) H_{2}(-\boldsymbol{p}) \\
& +Z_{1}\left(\boldsymbol{p}^{2}\right) Z_{2}\left(\boldsymbol{p}^{2}\right)\left[\Lambda_{1}^{+}(\boldsymbol{p}) \gamma_{0} I(\boldsymbol{p}) \Lambda_{2}^{-}(\boldsymbol{p}) \gamma_{0}-\Lambda_{1}^{-}(\boldsymbol{p}) \gamma_{0} I(\boldsymbol{p}) \Lambda_{2}^{+}(\boldsymbol{p}) \gamma_{0}\right] \\
& =M_{\mathrm{B}} \Phi(\boldsymbol{p}) .
\end{aligned}
$$

\subsection{Slightly Alternate Definition of Bethe-Salpeter Amplitude}

In order to make contact with some notation adopted in investigations of the instantaneous Bethe-Salpeter equation presented in Refs. $[6,7,17,18]$ which construct the Bethe-Salpeter amplitude from the matrix element $\left\langle 0\left|\mathrm{~T}\left(\psi_{1}\left(x_{1}\right) \psi_{2}^{\dagger}\left(x_{2}\right)\right)\right| \mathrm{B}(P)\right\rangle$, i.e., using $\psi_{2}^{\dagger}(x)$ instead of $\bar{\psi}_{2}(x)$, we have to redefine both Salpeter amplitude and Bethe-Salpeter kernel according to

$$
\chi(\boldsymbol{p}) \equiv \Phi(\boldsymbol{p}) \gamma_{0}, \quad W(\boldsymbol{p}, \boldsymbol{q}) \chi(\boldsymbol{q}) \equiv \gamma_{0} \hat{K}(\boldsymbol{p}, \boldsymbol{q}) \Phi(\boldsymbol{q})
$$

and, for convenience, we introduce for the instantaneous interaction term the abbreviation

$$
J(\boldsymbol{p}) \equiv \gamma_{0} I(\boldsymbol{p})=\frac{1}{(2 \pi)^{3}} \int \mathrm{d}^{3} q W(\boldsymbol{p}, \boldsymbol{q}) \chi(\boldsymbol{q})
$$

With this, we obtain the generalization, to the case of exact propagators of the bound-state constituents, of the eigenvalue form of the Salpeter equation recalled in Refs. $[6,8,9,14,17]$ :

$$
\begin{aligned}
& H_{1}(\boldsymbol{p}) \chi(\boldsymbol{p})-\chi(\boldsymbol{p}) H_{2}(\boldsymbol{p})+Z_{1}\left(\boldsymbol{p}^{2}\right) Z_{2}\left(\boldsymbol{p}^{2}\right)\left[\Lambda_{1}^{+}(\boldsymbol{p}) J(\boldsymbol{p}) \Lambda_{2}^{-}(\boldsymbol{p})-\Lambda_{1}^{-}(\boldsymbol{p}) J(\boldsymbol{p}) \Lambda_{2}^{+}(\boldsymbol{p})\right] \\
& =M_{\mathrm{B}} \chi(\boldsymbol{p}) .
\end{aligned}
$$

As a trivial observation, the redefinition (12) of Salpeter amplitude and Bethe-Salpeter kernel must be taken into account in the discussion of the Lorentz (or spin) structure of the interaction kernel. Suppose that the kernel $\hat{K}(\boldsymbol{p}, \boldsymbol{q})$ acts on the Salpeter amplitude $\Phi(\boldsymbol{p})$ as

$$
\hat{K}(\boldsymbol{p}, \boldsymbol{q}) \Phi(\boldsymbol{q})=V(\boldsymbol{p}, \boldsymbol{q}) \Gamma \Phi(\boldsymbol{q}) \Gamma
$$

with some Lorentz-scalar interaction function $V(\boldsymbol{p}, \boldsymbol{q})$ and generic Dirac matrices $\Gamma$. In this case, the kernel $\hat{K}(\boldsymbol{p}, \boldsymbol{q})$ is said to be of Lorentz structure $\Gamma \otimes \Gamma$ and written symbolically in the form of the product of the interaction function $V(\boldsymbol{p}, \boldsymbol{q})$ and the tensor product $\Gamma \otimes \Gamma$ :

$$
\hat{K}(\boldsymbol{p}, \boldsymbol{q})=V(\boldsymbol{p}, \boldsymbol{q}) \Gamma \otimes \Gamma .
$$

The redefined interaction kernel $W(\boldsymbol{p}, \boldsymbol{q})$ acts on the redefined Salpeter amplitude $\chi(\boldsymbol{p})$ as

$$
W(\boldsymbol{p}, \boldsymbol{q}) \chi(\boldsymbol{q})=V(\boldsymbol{p}, \boldsymbol{q}) \tilde{\Gamma} \chi(\boldsymbol{q}) \tilde{\Gamma},
$$

where the Lorentz structure $\tilde{\Gamma} \otimes \tilde{\Gamma}$ of the redefined kernel $W(\boldsymbol{p}, \boldsymbol{q})=V(\boldsymbol{p}, \boldsymbol{q}) \tilde{\Gamma} \otimes \tilde{\Gamma}$ is related to its counterpart in the original kernel $\hat{K}(\boldsymbol{p}, \boldsymbol{q})$, i.e., $\Gamma \otimes \Gamma$, according to $\tilde{\Gamma} \otimes \tilde{\Gamma}=\gamma_{0} \Gamma \otimes \gamma_{0} \Gamma$. 


\subsection{Salpeter Equation}

In addition to the instantaneous approximation (3), the derivation of the Salpeter equation [2] invokes the further assumption that the exact fermion propagators $S_{i}(p)$ entering in the Bethe-Salpeter equations (1) or (2) may be reasonably approximated by the corresponding free propagators (6), that is, $S_{i}(p)=S_{0}\left(p, m_{i}\right)$. In this approximation the mass parameters $m_{i}$ in the free propagators are then interpreted as some effective (or "constituent") masses of the fermionic bound-state constituents. Accordingly the Salpeter equation may be easily recovered from the more general instantaneous Bethe-Salpeter formalism developed in this analysis by performing, for the exact functions $M_{i}\left(p^{2}\right)$ and $Z_{i}\left(p^{2}\right)$, the free-propagator limit

$$
M_{i}\left(p^{2}\right) \rightarrow m_{i}, \quad Z_{i}\left(p^{2}\right) \rightarrow 1 .
$$

With our definitions of energies $E_{i}(\boldsymbol{p})$ and Hamiltonians $H_{i}(\boldsymbol{p})$ in Subsect. 2.4, this implies

$$
E_{i}(\boldsymbol{p}) \rightarrow \sqrt{\boldsymbol{p}^{2}+m_{i}^{2}}, \quad H_{i}(\boldsymbol{p}) \rightarrow \gamma_{0}\left(\boldsymbol{\gamma} \cdot \boldsymbol{p}+m_{i}\right) .
$$

Other choices for the effective propagators of the free (i.e., non-interacting) fermions in the instantaneous Bethe-Salpeter equation (4) entail different three-dimensional reductions of the Bethe-Salpeter equation. For comparisons of the various proposals, see Refs. [47-50].

\section{Summary, Conclusions, and Outlook}

We analyzed the instantaneous limit of the Bethe-Salpeter formalism and, by applying this approximation not only to the interaction kernel but also to the propagators of the involved bound-state constituents, we formulated, for the case of the exact (or full) propagators, the instantaneous Bethe-Salpeter equation for fermion-antifermion bound states (8) as well as its representation as eigenvalue problem in both arbitrary reference frame (10) and the rest frame of the bound state (11). Retaining (an approximation to) the full fermion propagator should facilitate the embedding of a mechanism for spontaneous chiral symmetry breaking. All exact propagators required may be extracted, with due care, from lattice computations. Our findings thus may improve the understanding of various bound systems; corresponding studies are in progress [51]. Clearly, the generalization of our results to systems involving as bound-state constituents also particles other than spin- $\frac{1}{2}$ fermions runs along similar lines.

\section{Acknowledgements}

One of us (W. L.) would like to thank Craig D. Roberts for a lot of stimulating discussions.

\section{References}

[1] E. E. Salpeter and H. A. Bethe, Phys. Rev. 84 (1951) 1232.

[2] E. E. Salpeter, Phys. Rev. 87 (1952) 328.

[3] W. Lucha, F. F. Schöberl, and D. Gromes, Phys. Rep. 200 (1991) 127.

[4] W. Lucha and F. F. Schöberl, Int. J. Mod. Phys. A 7 (1992) 6431. 
[5] A. Le Yaouanc, L. Oliver, S. Ono, O. Pène, and J.-C. Raynal, Phys. Rev. D 31 (1985) 137.

[6] J.-F. Lagaë, Phys. Rev. D 45 (1992) 305.

[7] J.-F. Lagaë, Phys. Rev. D 45 (1992) 317.

[8] J. Resag, C. R. Münz, B. C. Metsch, and H. R. Petry, Nucl. Phys. A 578 (1994) 397, nucl-th/9307026;

J. Resag, Analysis of the instantaneous Bethe-Salpeter equation and its application to q $\bar{q}$ bound states, Ph. D thesis, University of Bonn (1994).

[9] C. R. Münz, J. Resag, B. C. Metsch, and H. R. Petry, Nucl. Phys. A 578 (1994) 418, nucl-th/9307027;

C. R. Münz, Meson decays and form factors in a relativistic quark model, Ph. D thesis, University of Bonn (1994).

[10] C. R. Münz, J. Resag, B. C. Metsch, and H. R. Petry, Phys. Rev. C 52 (1995) 2110, nucl-th/9406035.

[11] J. Resag and C. R. Münz, Nucl. Phys. A 590 (1995) 735, nucl-th/9407033.

[12] G. Zöller, S. Hainzl, C. R. Münz, and M. Beyer, Z. Phys. C 68 (1995) 103, hep-ph/9412355.

[13] E. Klempt, B. C. Metsch, C. R. Münz, and H. R. Petry, Phys. Lett. B 361 (1995) 160, hep-ph/9507449.

[14] C. R. Münz, Nucl. Phys. A 609 (1996) 364, hep-ph/9601206.

[15] J. Parramore and J. Piekarewicz, Nucl. Phys. A 585 (1995) 705, nucl-th/9402019.

[16] J. Parramore, H.-C. Jean, and J. Piekarewicz, Phys. Rev. C 53 (1996) 2449, nuclth/9510024.

[17] M. G. Olsson, S. Veseli, and K. Williams, Phys. Rev. D 52 (1995) 5141, hep-ph/9503477.

[18] M. G. Olsson, S. Veseli, and K. Williams, Phys. Rev. D 53 (1996) 504, hep-ph/9504221.

[19] M. Koll, R. Ricken, D. Merten, B. C. Metsch, and H. R. Petry, Eur. Phys. J. A 9 (2000) 73, hep-ph/0008220;

M. Koll, Electroweak processes with light mesons in a relativistic quark model, $\mathrm{Ph}$. D thesis, University of Bonn (2001).

[20] R. Ricken, M. Koll, D. Merten, B. Ch. Metsch, and H. R. Petry, Eur. Phys. J. A 9 (2000) 221, hep-ph/0008221;

R. Ricken, Properties of light mesons in a relativistic quark model, Ph. D thesis, University of Bonn (2001).

[21] D. Merten, R. Ricken, M. Koll, B. Metsch, and H. Petry, Eur. Phys. J. A 13 (2002) 477, hep-ph/0104029;

D. Merten, Hadron form factors and decays, Ph. D thesis, University of Bonn (2002).

[22] R. Ricken, M. Koll, D. Merten, and B. Ch. Metsch, Eur. Phys. J. A 18 (2003) 667, hep$\mathrm{ph} / 0302124$.

[23] W. Lucha, K. Maung Maung, and F. F. Schöberl, Phys. Rev. D 63 (2001) 056002, hep$\mathrm{ph} / 0009185$. 
[24] W. Lucha, K. Maung Maung, and F. F. Schöberl, in: Proc. Int. Conf. on Quark Confinement and the Hadron Spectrum IV, edited by W. Lucha and K. Maung Maung (World Scientific, New Jersey/London/Singapore/Hong Kong, 2002), p. 340, hep-ph/0010078.

[25] W. Lucha, K. Maung Maung, and F. F. Schöberl, Phys. Rev. D 64 (2001) 036007, hep$\mathrm{ph} / 0011235$.

[26] W. Lucha and F. F. Schöberl, Int. J. Mod. Phys. A 17 (2002) 2233, hep-ph/0109165.

[27] M. Böhm, Nucl. Phys. B 91 (1975) 494.

[28] C. Alabiso and G. Schierholz, Nucl. Phys. B 110 (1976) 81.

[29] C. Alabiso and G. Schierholz, Nucl. Phys. B 126 (1977) 461.

[30] H. Pagels, Phys. Rev. D 15 (1977) 2991.

[31] T. Hofsäss and G. Schierholz, Phys. Lett. B 76 (1978) 125.

[32] R. Roth, Nucl. Phys. B 154 (1979) 21.

[33] C. D. Roberts and A. G. Williams, Prog. Part. Nucl. Phys. 33 (1994) 477, hep-ph/9403224.

[34] C. D. Roberts and S. M. Schmidt, Prog. Part. Nucl. Phys. 45 (2000) S1, nucl-th/0005064.

[35] C. D. Roberts, nucl-th/0007054.

[36] R. Alkofer and L. von Smekal, Phys. Rep. 353 (2001) 281, hep-ph/0007355.

[37] P. Maris, A. Raya, C. D. Roberts, and S. M. Schmidt, Eur. Phys. J. A 18 (2003) 231, nucl-th/0208071.

[38] P. Maris and C. D. Roberts, Int. J. Mod. Phys. E 12 (2003) 297, nucl-th/0301049.

[39] C. D. Roberts, Lect. Notes Phys. 647 (2004) 149, nucl-th/0304050.

[40] J. R. Finger and J. E. Mandula, Nucl. Phys. B 199 (1982) 168.

[41] A. Le Yaouanc, L. Oliver, O. Pène, and J.-C. Raynal, Phys. Rev. D 29 (1984) 1233.

[42] S. L. Adler and A. C. Davis, Nucl. Phys. B 244 (1984) 469.

[43] A. Kocić, Phys. Rev. D 33 (1986) 1785.

[44] R. Alkofer and P. A. Amundsen, Nucl. Phys. B 306 (1988) 305.

[45] S. P. Klevansky and R. H. Lemmer, Phys. Rev. D 38 (1988) 3559.

[46] R. Alkofer, P. A. Amundsen, and K. Langfeld, Z. Phys. C 42 (1989) 199.

[47] T. Babutsidze, T. Kopaleishvili, and A. Rusetsky, Phys. Lett. B 426 (1998) 139, hep$\mathrm{ph} / 9710278$.

[48] T. Babutsidze, T. Kopaleishvili, and A. Rusetsky, Phys. Rev. C 59 (1999) 976, hepph/9807485.

[49] T. Kopaleishvili, Phys. Part. Nucl. 32 (2001) 560, hep-ph/0101271. 
[50] T. Babutsidze, T. Kopaleishvili, and D. Kurashvili, Georgian Electronic Scientific J.: Phys. 1 (2004) 20, hep-ph/0308072.

[51] Li Z.-F., W. Lucha, and F. F. Schöberl, in preparation. 